\title{
Ab Initio 3-D Electron Crystallographic Determination of a Zeolite Structure by Maximum Entropy and Likelihood
}

\author{
D. L. Dorset*, and C. J. Gilmore** \\ *ExxonMobil Res \& Eng Co., Corporate Strategic Research, Route 22 East, Annandale, NJ 08801 \\ ${ }^{* *}$ Chemistry Department, University of Glasgow, Glasgow G12 8QQ Scotland, UK
}

The MWW family of zeolites, including MCM-22, MCM-49, and ITQ-1, may be represented by a common framework structure, differing only in $\mathrm{SiO}_{2} / \mathrm{Al}_{2} \mathrm{O}_{3}$ ratios. Within the $\pm 60^{\circ}$ tilt limits of a goniometer stage, 155 unique electron diffraction intensity data have been collected at $300 \mathrm{kV}$ (FEl/Philips CM30) from these representative materials in space group $P 6 / \mathrm{mmm}$, where $a=14.21, c=24.94 \AA$. Intensity data from individual samples cannot be distinguished from one another in three dimensions (owing partly to similar Al and Si scattering factors), correcting an earlier notion ${ }^{1}$, based solely on hk0 patterns, that subtle differences might occur, e. $\mathrm{g}$ between calcined MCM22 and MCM-49, prepared by different synthetic routes.

The crystal structure of MCM-22 was the first zeolite to be determined from electron diffraction intensities by direct phasing methods ${ }^{2}$. Although the data sampling was somewhat sparse, inclusion of rarely observed data, sampling the $c^{*}$ axis, seemed to facilitate the analysis. Preliminary zonal analyses of data from very thin MWW crystals ${ }^{1}$ revealed that the intensities are particularly favorable for structure analysis prompting the application of direct analysis to a more complete 3-D set.

Extension of 2-D phase solutions by conventional methods into 3-D, e. g. by the Sayre equation, was not very satisfactory. Adding one permissible origin-defining phase, phase solutions were sought by permutation for 16 strong reflections by the maximum entropy and likelihood procedure ${ }^{4}$ with the program package $\mathrm{MICE}^{5}$, evaluating solutions by a log likelihood gain FOM. A Nordström-Robinson error correcting code ${ }^{3}$ was used to reduce the phase permutation of 16 generator reflections from $2^{16}$ to 256 , accepting a small number of incorrect phases. An optimal solution was found at the 26th ranked phase set within 256 trials. (Most false solutions can be identified readily by inspection of the potential maps. A somewhat successful phase set was also identified at the 20th ranked trial.) This phase solution for hk0 phases gave a mean phase error of $45^{\circ}$, so that all tetrahedral sites and oxygen connectors could be located in the [001] map. The phase error for hk $\ell$ data was $53^{\circ}$ for the 27 most intense reflections. Owing to the well-known 'missing-cone problem' the potential function served only as an envelope for the structural framework, with density elongated along the c-axis direction. Some improvement of the 3-D map could be found when the unknown data were predicted by a Sayre extension of MICE generated 3-D phases. A somewhat distorted zeolite framework could be constructed directly from the density map that could be optimized dimensionally into the known framework structure (Fig. 1). For completely unambiguous structure analyses, methods must be found to acquire data within the missing cone region, suggesting samples prepared by focussed ion beam methods. 
References:

1. D. L. Dorset, Z. Kristallogr. 218 (2003) 612.

2. S. Nicolopoulos, et. al., J. Amer. Chem. Soc. 117 (1995) 8947.

3. C. Gilmore, et al., Acta Crystallogr. A55 (1999) 70.

4. G. Bricogne and C. J. Gilmore, Acta Crystallogr. A46 (1990) 284.

5. C. J. Gilmore, et al., Trans. Amer. Cryst. Assoc. 30 (1994) 15.

6. M. A. Camblor, et al, J. Phys. Chem. B102 (1998) 44.
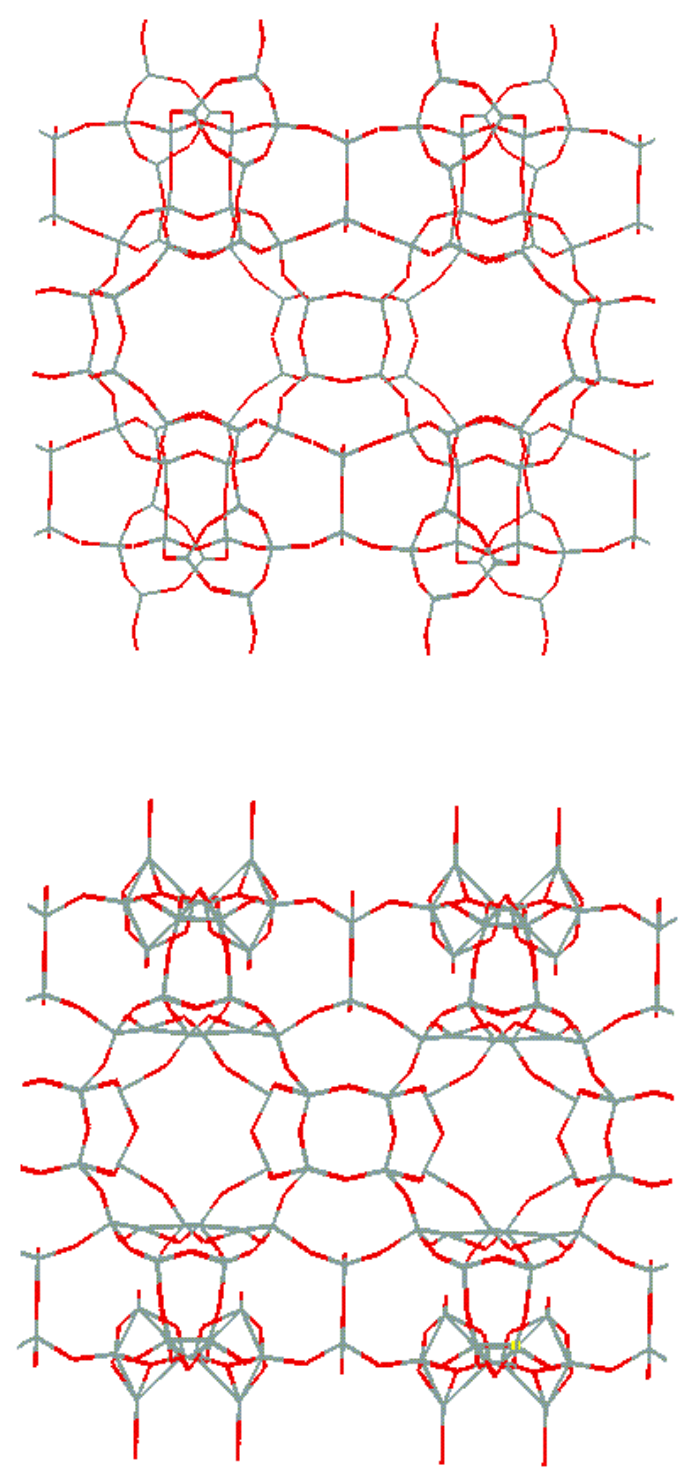

Figure 1. (top) ideal ITQ-1 framework ${ }^{6}$; (bottom) direct electron crystallographic structure determination. 